\title{
The 8-tetrahedra longest-edge partition of right-type tetrahedra
}

\author{
A. Plaza ${ }^{\text {a } *}$, M.A. Padrón ${ }^{\mathrm{b}}$, J.P. Suárez ${ }^{\mathrm{c}}$, S. Falcón ${ }^{\mathrm{a}}$ \\ ${ }^{a}$ Department of Mathematics, Universidad de Las Palmas de Gran Canaria (ULPGC), Edif. Informatica y Matematicas, \\ Las Palmas de Gran Canaria, 35017, Spain \\ ${ }^{\mathrm{b}}$ Department of Civil Engineering, ULPGC, Spain \\ ${ }^{\mathrm{c}}$ Department of Cartography and Graphic Engineering, ULPGC, Spain
}

Received 29 June 2003; received in revised form 18 March 2004; accepted 11 April 2004

\begin{abstract}
A tetrahedron $t$ is said to be a right-type tetrahedron, if its four faces are right triangles. For any right-type initial tetrahedron $t$, the iterative 8-tetrahedra longest-edge partition of $t$ yields into a sequence of right-type tetrahedra. At most only three dissimilar tetrahedra are generated and hence the non-degeneracy of the meshes is simply proved. These meshes are of acute type and then satisfy trivially the maximum angle condition. All these properties are highly favorable in finite element analysis. Furthermore, since a right prism can be subdivided into six right-type tetrahedra, the combination of hexahedral meshes and right tetrahedral meshes is straightforward.
\end{abstract}

(C) 2004 Elsevier B.V. All rights reserved.

Keywords: 8-tetrahedra longest-edge partition; Right-type tetrahedron; Maximum angle condition; Non-degeneracy; Similarity classes

\section{Introduction}

The development of automatic adaptive finite element analysis procedure has received much attention since such programs allow the user to obtain finite element solutions for many engineering problems within prescribed accuracy [1,2], for example in fluid mechanic problems, or for determining the temperature distribution or the stress field. An adaptive finite element program performs, in sequence, the finite element analysis, error estimation, and mesh generation. This cycle has to be repeated until the prescribed accuracy is achieved. The objective of an adaptive refinement program is to control the discretization error by

\footnotetext{
* Corresponding author. Tel.: 34928458827; fax: 34928458811.

E-mail address: aplaza@dmat.ulpgc.es (A. Plaza).
} 
increasing the number of degrees of freedom in regions where the previous finite element model presents higher error. It should be noted that there are many difficulties in generating a three-dimensional (3D) mesh for an arbitrary object of complicated geometrical shape in compliance with a specified node spacing function $[3,4]$.

A major class of refinement methods is based on the simplex bisection. Among the bisection-based partitions most commonly used in two dimensions, is the Newest Vertex Bisection presented by Mitchell [5]. Only four similarity classes of triangles and only eight distinct angles are created by this method and, thus, the angles satisfy the important condition of being bounded away from 0 and $\pi$. As noted by Babuška and Aziz [6] for triangular meshes, when the maximum angle approaches $\pi$, the interpolation error grows. The maximum angle condition has also been generalized to tetrahedral elements by Kř́ı̌žek [7].

Another well-known partition is the 4-Triangles Longest-Edge (4T-LE) partition introduced and studied by Rivara [8]. The 4T-LE partition presents suitable properties in its application for solving problems with the Finite Element method such as the property of self-improvement, non-degeneracy and locality of the refinement. It should be noted that in the case of a right-angled initial triangle $t_{0}$ all the triangles generated by the 4T-LE partition are similar to $t_{0}$. In this sense, it can be said that the right-angled triangle class is the class of regular triangles for this partition.

The 4T-LE partition and associated local refinement has been extended to three dimensions recently [9]. However the validity of the self-improvement and non-degeneracy properties of the 2D remain to be proved for the 3D case.

We focus in this paper on the 8-tetrahedra longest-edge (8T-LE) partition of a special type of tetrahedra, called right-type tetrahedra. These tetrahedra, analogous to the right triangles in two dimensions, have four right triangles as faces. The study of the partition of these tetrahedra is of interest because the conversion from a octree-based hexahedral mesh [10] to a tetrahedral mesh is straightforward [11]. By assuring good properties as to a low number of similarity classes and non-degeneracy for these tetrahedra is also a first step in the study of the 8T-LE partition and associated refinement to more general tetrahedral elements.

For any right-type initial tetrahedron $t$, we prove that the iterative 8T-LE partition of $t$ yields into a sequence of right-type tetrahedra. At most only three dissimilar tetrahedra are generated and hence the non-degeneracy of the meshes is simply proved. These meshes are of acute type and then satisfy trivially the maximum angle condition. All these properties are highly favorable in finite element analysis.

\section{Basic definitions and preliminaries}

Definition 1 (Simplex). A closed subset $T \subset \mathbb{R}^{n}$ is called a $(k)$-simplex, $0 \leqslant k \leqslant n$ if $T$ is the convex linear hull of $k+1$ vertices $\mathbf{x}^{(0)}, \mathbf{x}^{(1)}, \ldots, \mathbf{x}^{(k)} \in \mathbb{R}^{n}$, and it will be denoted by $T=\left[\mathbf{x}^{(0)}, \mathbf{x}^{(1)}, \ldots, \mathbf{x}^{(k)}\right]$.

If $k=n$ then $T$ is called simplex in $\mathbb{R}^{n}$. In what follows (2)-simplices and (3)-simplices are also called triangles and tetrahedra, respectively.

Definition 2 (Similar simplices). Two simplices $t, t^{\prime} \subset \mathbb{R}^{n}$ are called similar to each other if there exists a translation vector $\mathbf{a} \in \mathbb{R}^{n}$, a scaling factor $c>0$, and an orthogonal matrix $\mathbf{Q} \in \mathbb{R}^{n \times n}$ such that

$$
t^{\prime}=\mathbf{a}+c \mathbf{Q} t
$$


Symbol "=" in Eq. (1) must be understood in the sense of sets, so the similarity class of a simplex is independent of its vertex ordering. Note that two similar simplices are equivalent under translation, scaling, rotation and mirror reflection. The similarity relation is an equivalence relation. The set of similar simplices to any given simplex $t$ is its similarity class.

Definition 3 (Conforming triangulation). Let $\Omega$ be any bounded domain in $\mathbb{R}^{2}$ or $\mathbb{R}^{3}$ with non-empty interior and polygonal boundary $\partial \Omega$, and consider a partition of $\Omega$ into a set of triangles $\tau=\left\{t_{1}, t_{2}, t_{3}, \ldots, t_{n}\right\}$. Then we say that $\tau$ is a conforming triangulation if the following properties hold:

(1) $\Omega=\bigcup t_{i}$;

(2) interior $\left(t_{i}\right) \neq \emptyset, \forall t_{i} \in \tau$;

(3) interior $\left(t_{i}\right) \bigcap$ interior $\left(t_{j}\right)=\emptyset$, if $i \neq j$;

(4) $\forall t_{i}, t_{j} \in \tau$ with $t_{i} \cap t_{j} \neq \emptyset$, then $t_{i} \cap t_{j}$ is an entire face or a common edge, or a common vertex.

Definition 4. The 4T-LE partition of a triangle $t$ is obtained by joining the midpoint of the longest edge of $t$ with the opposite vertex and with the midpoints of the two remaining edges.

Note that a triangle $t$ may have a non-unique longest edge. In this case the longest-edge is chosen from among one of the longest-edges in order to minimize the extension of the conformity area.

Based on [12] it has been proved that the longest-edge based partitions verify the following property of non-degeneracy: The iterative use of these partitions over any initial triangulation only produces triangles whose smallest interior angles are always greater than or equal to $\alpha / 2$, where $\alpha$ is the smallest interior angle of the initial triangulation.

For the 4T-LE partition, the number of similarity classes of triangles generated has been proved to be finite but this number depends on the geometry of the initial triangle [13,14]. Furthermore, the application of the 4T-LE partition shows a self-improvement property $[8,14]$ in the sense that the application to any obtuse triangle $t_{0}$ of the 4T-LE partition produces a unique distinct couple of similar triangles $t_{1}$, whose 4T-LE partition in turn produces a new distinct couple of similar triangles $t_{2}$, and so on, until a last non-obtuse triangle $t_{n}$ is obtained. Moreover, the smallest angle increases and the largest angle decreases until the first non-obtuse triangle is obtained.

In three dimensions, several techniques have been developed in recent years for refining (and coarsening) tetrahedral meshes by means of bisection. On the contrary to the $2 \mathrm{D}$ case, the non-degeneracy of 3D longest-edge bisection based partitions is still an open problem. Liu and Joe offer one of the approaches based on bisection [15]. This partition can be understood as the 3D version of the Mitchell partition. The edges for bisection are chosen without any computation following a rule between the edge types involved and their relative position to automatically assign the types to the new edges [15].

Recently, a partition in eight tetrahedra based on the length of the edges, the 8T-LE partition, has been investigated and used for local refining and coarsening tetrahedral meshes $[9,16]$. The 8T-LE partition can be achieved by performing a sequence of bisections through the midpoints of the edges of the original tetrahedron taking into account the length of the edges as follows [17]:

Definition 5. For any tetrahedron $t$ of unique longest-edge (primary edge), the primary faces of $t$ are the two faces of $t$ that share the longest-edge of $t$. In addition, the two remaining faces of $t$ are called 


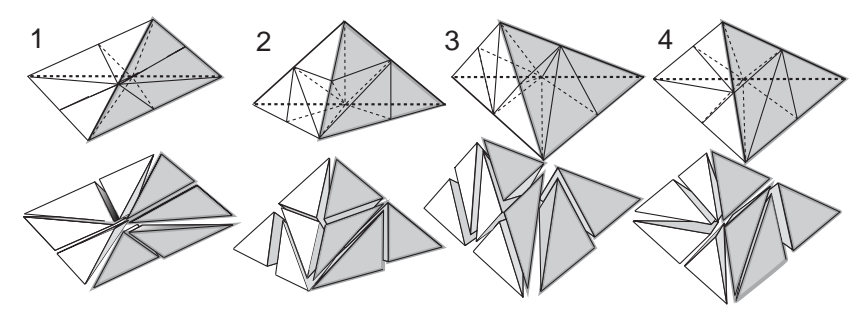

Fig. 1. The four refinement patterns for the 8T-LE. The primary edge is indicated with a dashed bold line, the secondary edges in bold.

secondary faces of $t$. Furthermore, the secondary edges of $t$ are the longest edges of the secondary faces of $t$ ( 1 or 2 secondary longest edges). The remaining edges of $t$ are called tertiary edges.

For any tetrahedron $t$ of unique longest-edge, the primary faces of $t$ have a common longest-edge equal to the longest-edge of $t$. In order to avoid ambiguousness, if tetrahedron $t$ has a unique longest-edge, we first choose the longest-edge as primary edge and then the two primary faces are chosen as the faces sharing the primary edge. For each tetrahedron $t$ having a non-unique longest-edge, the primary edge is chosen in order to minimize the extension of the refinement for the conformity of the mesh. In a similar way a unique selection for secondary edges is performed in a consistent manner with the faces. These selections are consistently maintained throughout the overall refinement process.

Definition 6. For any tetrahedron $t$ of unique longest-edge and unique secondary edges, the 8T-LE partition of $t$ is defined as follows:

(1) longest edge bisection of $t$ producing tetrahedra $t_{1}, t_{2}$;

(2) bisection of $t_{i}$ by the midpoint of the unique edge of $t_{i}$ which is also a secondary edge of $t$, producing tetrahedra $t_{i j}$ for $i, j=1,2$;

(3) bisection of each $t_{i j}$ by the midpoint of the unique edge equal to a tertiary edge of $t$, for $i, j=1,2$.

Theorem 7 (Plaza and Rivara [17]). The 8-tetrahedra longest-edge partition of any tetrahedron t produces both a conforming volume triangulation of t and a conforming surface triangulation of $t$ such that:

(1) The conforming surface triangulation of $t$ is identical to the surface triangulation obtained by the 4-triangles partition of the faces of $t$.

(2) Four different triangulation patterns are obtained (Fig. 1) according to the relative position of the longest-edge and the secondary edges of t. Each one of these four patterns produces only one new internal edge (connecting the midpoint of the longest-edge of t, and the midpoint of the edge opposite to the longest-edge) and eight new internal faces.

For any tetrahedron $t$, the 8T-LE partition of $t$ produces eight sub-tetrahedra by performing the 4T-LE partition of the faces of $t$, and by subdividing the interior of the tetrahedron $t$ consistently with the division of the faces (see Fig. 1).

Under the assumption that the longest-edge and the secondary edges are unique, there is, likewise, a unique correspondence between the four volume partition patterns produced by the 8 -tetrahedra partition 
of any tetrahedron $t$ and the four surface partition patterns obtained by the 4-triangles partition of the faces of $t$.

\section{The 8T-LE partition of right-type tetrahedra}

Consider an arbitrary tetrahedron $t$. Then each one of the six angles between any pair of its faces is called a dihedral interior angle or an interior angle. A tetrahedral partition is said to be of acute type if all six interior angles of any tetrahedron in the partition are less than or equal to $\pi / 2$. This definition was introduced by Korotov and Kř́̌žek in [18]. They also presented a technique to perform refinements on acute type tetrahedral partitions of a polyhedral domain, provided that the center of the circumscribed sphere around each tetrahedron belongs to the tetrahedron, and also used to prove the discrete maximum principle for nonlinear elliptic problems [19].

In this section, we study the 8T-LE partition of right-type tetrahedra. These tetrahedra are analogous to the right triangles in two dimensions. We shall prove that the iterative 8T-LE partition of $t$ yields into a sequence of right-type tetrahedra. At most, only three dissimilar tetrahedra are generated and, hence, the non-degeneracy of the meshes is simply proved. A tetrahedral triangulation in which all the tetrahedra are right-type is also of acute-type and, hence, the discrete maximum principle for nonlinear elliptic problems can be proved for such tetrahedral domains (see [19]). As noted in the Introduction these properties are to be desired in finite element analysis.

Definition 8 (Right-type tetrahedron). A tetrahedron $t$ is said to be a right-type tetrahedron, if its four faces are right triangles.

In a right-type tetrahedron $t$, there are three mutually perpendicular edges which do not pass through the same vertex, and are called legs of $t$. One of them has one vertex in common with each one of the other two legs. This leg is called the central leg and the others are the extreme legs. If the three legs are of the same length, the right-type tetrahedron will be called regular right tetrahedron. If only two legs have the same length, the tetrahedron will be called isosceles, and in other case, it will be called scalene right tetrahedron. The legs define, by their parallelism, a unique orthohedron $\mathscr{P}$ that we call orthohedron-hull of $t$, such that $t \subset \mathscr{P}$; the vertices of $t$ are also vertices of $\mathscr{P}$, and the longest edge of $t$ is an internal diagonal of $\mathscr{P}$. Note that the legs of a right-type tetrahedron are also legs of the four faces of the tetrahedron. Moreover, the length and relative position of the legs determine the shape of any right-type tetrahedron. Let $t$ be a right-type tetrahedron with legs $a, b$, and $c$, such that $b$ is between $a$ and $c$; then $t=t(a, b, c)$ will denote the tetrahedron $t$ and, likewise, the class of the similar tetrahedra to $t,[t]$.

A picture of a right-type tetrahedron $t$ is presented in Fig. 2. The four faces of $t$ are right-angled triangles, where the legs are highlighted in Fig. 2(b). The edges apart from the legs are precisely the primary and secondary edges of $t$ (in bold in Fig. 2(a)).

The following property is a direct result of a tetrahedron being the closed convex hull of its vertices, from Definition 2, and the fact that the extreme points of the legs of a right-type tetrahedron determine its four vertices. 


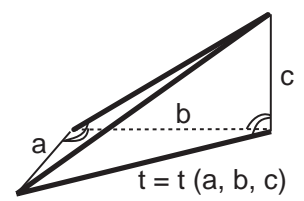

(a)

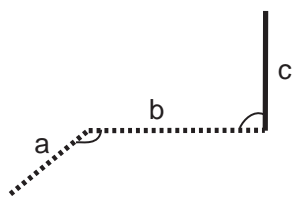

(b)

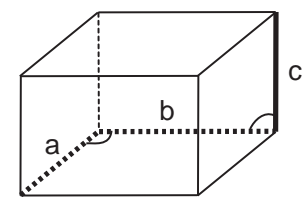

(c)

Fig. 2. Right-type tetrahedron $t=t(a, b, c)$, legs and associated orthohedron $\mathscr{P}$.

Theorem 9. Two right-type tetrahedra $t(a, b, c)$ and $t^{\prime}\left(a^{\prime}, b^{\prime}, c^{\prime}\right)$ are similar to each other if and only if their extreme legs are in the same ratio as their central legs. That is, either $b / b^{\prime}=a / a^{\prime}=c / c^{\prime}$, or $b / b^{\prime}=a / c^{\prime}=c / a^{\prime}$.

The 8T-LE partition of a right-type tetrahedron $t$ can be described as a function of its vertices. To this objective, consider $t=\left[\mathbf{x}^{(0)}, \mathbf{x}^{(1)}, \mathbf{x}^{(2)}, \mathbf{x}^{(3)}\right]$, such that $\mathbf{x}^{(0)} \mathbf{x}^{(1)}, \mathbf{x}^{(1)} \mathbf{x}^{(2)}$, and $\mathbf{x}^{(2)} \mathbf{x}^{(3)}$ are the legs of $t$. Observe that, the primary edge of $t$ is $\mathbf{x}^{(0)} \mathbf{x}^{(3)}$. For $0 \leqslant i, j \leqslant 3, i \neq j$ we denote $\mathbf{x}^{(i j)}:=\left(\mathbf{x}^{(i)}+\mathbf{x}^{(j)}\right) / 2$ the edge midpoint of $\mathbf{x}^{(i)} \mathbf{x}^{(j)}$. The 8T-LE partition of $t$ can be formulated as follows:

\section{Algorithm 8T-LE partition of right-tetrahedron (t)}

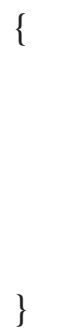

$$
\begin{array}{rlrl}
\text { divide } t & =\left[\mathbf{x}^{(0)}, \mathbf{x}^{(1)}, \mathbf{x}^{(2)}, \mathbf{x}^{(3)}\right] \text { into subtetrahedra } t_{i}, 1 \leqslant i \leqslant 8, \text { given by } \\
t_{1}: & :=\left[\mathbf{x}^{(0)}, \mathbf{x}^{(01)}, \mathbf{x}^{(02)}, \mathbf{x}^{(03)}\right], & t_{5}:=\left[\mathbf{x}^{(2)}, \mathbf{x}^{(02)}, \mathbf{x}^{(12)}, \mathbf{x}^{(03)}\right], \\
t_{2}: & :=\left[\mathbf{x}^{(1)}, \mathbf{x}^{(01)}, \mathbf{x}^{(02)}, \mathbf{x}^{(03)}\right], & t_{6}:=\left[\mathbf{x}^{(2)}, \mathbf{x}^{(12)}, \mathbf{x}^{(03)}, \mathbf{x}^{(13)}\right], \\
t_{3}: & :=\left[\mathbf{x}^{(1)}, \mathbf{x}^{(02)}, \mathbf{x}^{(12)}, \mathbf{x}^{(03)}\right], & t_{7}:=\left[\mathbf{x}^{(2)}, \mathbf{x}^{(03)}, \mathbf{x}^{(13)}, \mathbf{x}^{(23)}\right], \\
t_{4}: & :=\left[\mathbf{x}^{(1)}, \mathbf{x}^{(12)}, \mathbf{x}^{(13)}, \mathbf{x}^{(03)}\right], & t_{8}:=\left[\mathbf{x}^{(3)}, \mathbf{x}^{(03)}, \mathbf{x}^{(13)}, \mathbf{x}^{(23)}\right] .
\end{array}
$$

Let $t$ be a right-type initial tetrahedron in which the 8T-LE partition is applied. The successive applications of the 8T-LE partition to $t$ and its successors yield into an infinite sequence of tetrahedral nested meshes $\tau^{1}, \tau^{2}, \tau^{3}, \ldots$.

Theorem 10. Let $t_{0}$ be a right-type tetrahedron. Then, after applying the 8T-LE partition to $t_{0}$, we obtain eight tetrahedra also of a right-type. In addition, at most only three similarity classes are obtained throughout the iterative application of the $8 T-L E$ partition to $t_{0}$.

Proof. Our proof is based merely on simple geometrical arguments. Fig. 3(a) shows a right-type tetrahedron $t_{0}(a, b, c)=\left[\mathbf{x}^{(0)}, \mathbf{x}^{(1)}, \mathbf{x}^{(2)}, \mathbf{x}^{(3)}\right]$. The 8T-LE partition is applied to $t_{0}$ in Fig. 3(b). Note that, by their parallelism, tetrahedra $t_{1}:=\left[\mathbf{x}^{(0)}, \mathbf{x}^{(01)}, \mathbf{x}^{(02)}, \mathbf{x}^{(03)}\right]$ and $t_{8}:=\left[\mathbf{x}^{(3)}, \mathbf{x}^{(03)}, \mathbf{x}^{(13)}, \mathbf{x}^{(23)}\right]$ are similar to $t_{0}$, that is $t_{1}, t_{8} \in\left[t_{0}\right]$. Once these tetrahedra are deleted Fig. 3(c) is obtained.

By mirror reflection by the planes passing through points $\mathbf{x}^{(01)}, \mathbf{x}^{(02)}, \mathbf{x}^{(03)}$ and $\mathbf{x}^{(03)}, \mathbf{x}^{(13)}, \mathbf{x}^{(23)}$, respectively, tetrahedra with vertices $\mathbf{x}^{(1)}$ and $\mathbf{x}^{(2)}$ and the three vertices defining the reflection plane are also similar to their respective mirror images, and, hence, to the original tetrahedron. Thus, four tetrahedra share the internal edge $\mathbf{x}^{(12)} \mathbf{x}^{(03)}$, see Fig. 3(d). The figure also shows that tetrahedra $t_{1}$ are similar to 


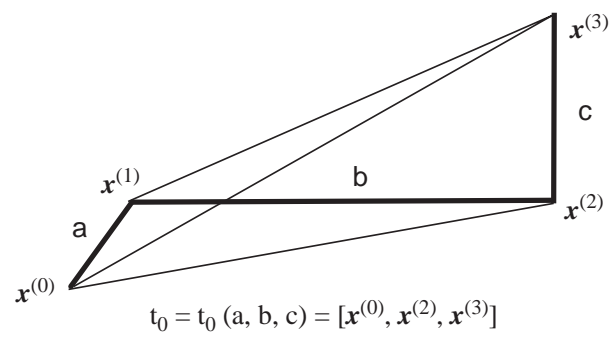

(a)

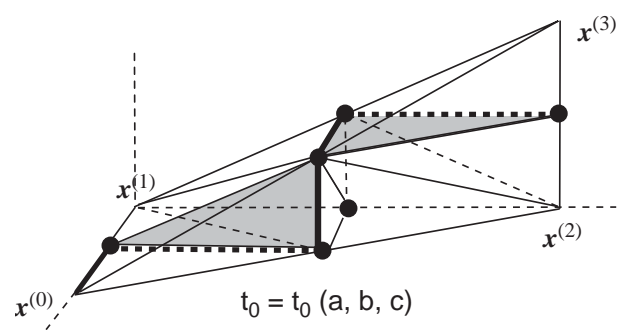

(b)

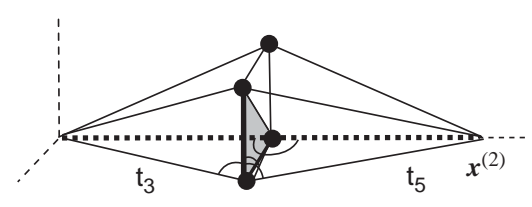

(d)

$$
t_{1}=t_{1}(b, a, c)
$$

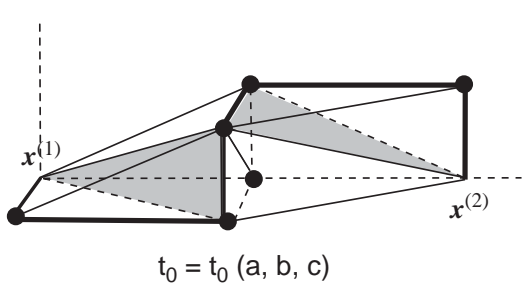

(c)

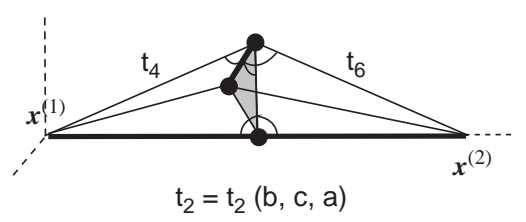

(e)

Fig. 3. 8T-LE partition of right-type tetrahedron into three classes of similar tetrahedra.

each other, but not necessarily similar to the initial tetrahedron. The marked right-angles indicate that the tetrahedra are right-type. Finally Fig. 3(e) shows the similarity between the last two tetrahedra $t_{2}$.

Based on Theorem 9, tetrahedra $t_{1}=t_{1}(b, a, c)$ and $t_{2}=t_{2}(a, c, b)$ in Fig. 3 belong to different classes. Note that the different possibilities of similarity classes generated, depend on the number of legs of different length of the initial tetrahedron. If they are of different lengths, we obtain four tetrahedra similar to the parent one, and two couples similar between them. If tetrahedron $t_{0}$ is isosceles, then we obtain two classes of tetrahedra, and, finally, if $t_{0}$ is regular, then all the tetrahedra obtained by the 8T-LE partition are similar to the former one.

From a right-type tetrahedron $t_{0}(a, b, c)$ only three similarity classes of right-type tetrahedra are obtained: $t_{0}(a, b, c), t_{1}=t_{1}(b, a, c)$ and $t_{2}=t_{2}(a, c, b)$. Since these classes are determined by the three different possibilities for the central leg, the 8T-LE partition is closed with respect to these three similarity classes, see Fig. 4.

Let $t_{i}^{(n)}$ be the number of tetrahedra belonging to the class $t_{i}$ for $i=0,1,2$ after $n$ applications of the 8T-LE partition to an initial right-type tetrahedron $t_{0}$. The recurrence relations associated to the 8T-LE 


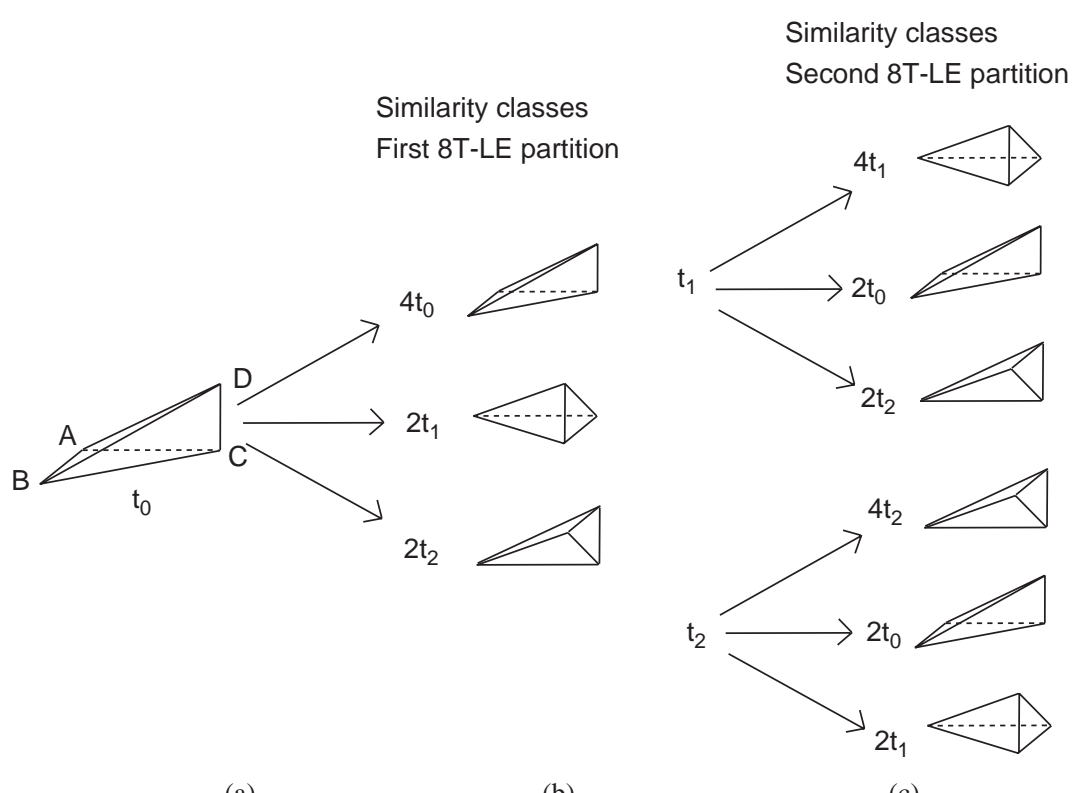

(a)

(b)

(c)

Fig. 4. Scheme of the generation of three similarity classes $t_{0}, t_{1}$ and $t_{2}$ by the 8T-LE partition of the tetrahedron $t_{0}$.

partition of an initial right-type tetrahedron $t_{0}$ are:

$$
\left\{\begin{array}{l}
t_{0}^{(n)}=4 t_{0}^{(n-1)}+2 t_{1}^{(n-1)}+2 t_{2}^{(n-1)} \\
t_{1}^{(n)}=2 t_{0}^{(n-1)}+4 t_{1}^{(n-1)}+2 t_{2}^{(n-1)} \\
t_{2}^{(n)}=2 t_{0}^{(n-1)}+2 t_{1}^{(n-1)}+4 t_{2}^{(n-1)}
\end{array}\right\} \text { for } n \geqslant 1
$$

with the initial conditions $t_{0}^{(0)}=1, t_{1}^{(0)}=0, t_{2}^{(0)}=0$.

Corollary 11. The 8T-LE partition of any right-type tetrahedron t does not degenerate.

Since the 8T-LE partition of a right-type tetrahedron $t_{0}$ only produces two other right-type tetrahedral similarity classes $t_{1}$ and $t_{2}$, it can be established, a priori, whether the partition of a right-type tetrahedron $t_{0}$ will improve the triangulation or not. The iterative application of equations (2) to a right scalene tetrahedron gives us the number of elements in each similarity class. The evolution of the percentage of the volume covered by each class is illustrated in Fig. 5.

Theorem 12. Let $t_{0}$ be a right-type tetrahedron. Then, after $n$ applications of the 8T-LE partition, the number of tetrahedra $t_{i}^{(n)}$ belonging to the class $t_{i}$ for $i=0,1,2$ are:

$$
t_{0}^{(n)}=\frac{8^{n}+2^{n+1}}{3}, \quad t_{1}^{(n)}=t_{2}^{(n)}=\frac{8^{n}-2^{n}}{3} .
$$




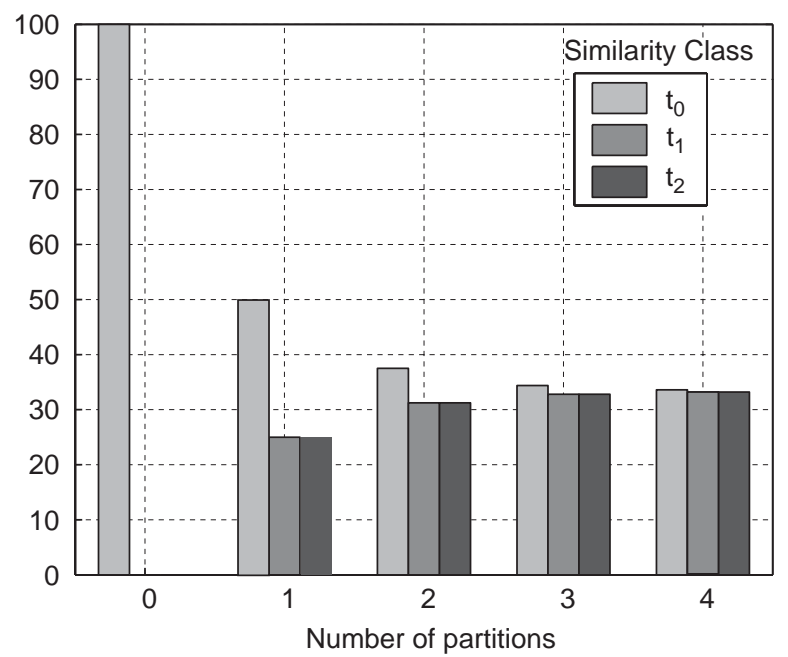

Fig. 5. Evolution of percentage of volume covered by each tetrahedral similarity class.

Proof. This proof is inductive. The formula given by the theorem is trivially true for $t_{0}^{(0)}$ since $t_{0}^{(0)}=1$. Let us suppose that the statement is true for $n=k-1$, then

$$
t_{0}^{(k-1)}=\frac{8^{k-1}+2^{k}}{3}, \quad t_{1}^{(k-1)}=t_{2}^{(k-1)}=\frac{8^{k-1}-2^{k-1}}{3} .
$$

By Eqs. (2), we have

$$
\begin{aligned}
t_{0}^{(k)} & =4 t_{0}^{(k-1)}+2 t_{1}^{(k-1)}+2 t_{2}^{(k-1)}=4 \frac{8^{k-1}+2^{k}}{3}+2 \frac{8^{k-1}-2^{k-1}}{3}+2 \frac{8^{k-1}-2^{k-1}}{3} \\
& =\frac{8^{k}+2^{k+1}}{3} .
\end{aligned}
$$

And, hence again from Eqs. (2) we get the result for $t_{1}^{(n)}$ and $t_{2}^{(n)}$.

Corollary 13. Let t $t_{0}$ be a right-type tetrahedron, and let $t_{0}, t_{1}$, and $t_{2}$ the three similarity classes produced by the 8T-LE partition when it is applied to $t_{0}$ and its successors. Then, when the number of global refinements $n$ tends to infinity, the volume covered by each class tends to cover one third of the initial tetrahedron volume.

It should be noted that the 8T-LE partition of a right-type tetrahedron is equivalent to the refinement technique presented in [18]. This partition is also equivalent to three levels of bisection refinement procedure applied to the DSS2 type tetrahedron in [15], and to the recursive approach proposed by Kossaczký in [20]. 


\section{Numerical example}

\subsection{Shape measures for tetrahedra}

We refer to Refs. [15,21-24] for the definitions, discussions and equations of different tetrahedron shape measures. A tetrahedron shape measure is a continuous function that evaluates the quality of a tetrahedron. It must be invariant under translation, rotation, reflection and uniform scaling of the tetrahedron, maximum for the regular tetrahedron and minimum for a degenerate tetrahedron. There should be no local maximum other than the global maximum for a regular tetrahedron and there should be no local minimum other than the global minimum for a degenerate tetrahedron [23].

As of here, we refer to some shape measures for tetrahedra. One of these measures is $\operatorname{ratio}(S)=$ $r(S) / R(S)$, where $r(S)$ is the length of the radius of the inscribed sphere in $s$ and $R(S)$ is the radius of the circumscribed sphere [25]).

For each vertex $P$ of a tetrahedron $t$, let us consider the value $\Phi_{P}$

$$
\Phi_{P}=\sin ^{-1}\left\{\left(1-\cos ^{2} \alpha_{P}-\cos ^{2} \beta_{P}-\cos ^{2} \gamma_{P}+2 \cos \alpha_{P} \cos \beta_{P} \cos \gamma_{P}\right)^{1 / 2}\right\},
$$

where $\alpha_{P}, \beta_{P}, \gamma_{P}$ are the associated corner angles at $P$. Then, we define the measure of tetrahedron $t$ as $\Phi_{t}=\min \left\{\Phi_{P}: P \in t\right\}$. The relation between $\Phi_{P}$ and the solid angle $\Omega_{P}$ at $P$ is (see $\left.[22,9]\right)$ :

$$
\Omega_{P}=2 \sin ^{-1} \frac{\sin \left(\Phi_{P}\right)}{4 \cos \left(\alpha_{P} / 2\right) \cos \left(\beta_{P} / 2\right) \cos \left(\gamma_{P} / 2\right)} .
$$

Liu and Joe $[15,21]$ introduced the estimate $\eta(t)=12(3 v)^{2 / 3} / \sum_{i=1}^{6} l_{i}^{2}$, where $v$ is the volume of $t$ and $l_{i}$ are the lengths of the edges of $t$. Since $\eta$ is the most economic in computation [26], it will be adopted here to judge the quality of an individual element.

\subsection{Local refinement example with different initial meshes}

We present the simulation of a 3D local refinement problem on a cubic domain through the local refinement algorithm associated to the 8T-LE partition $[9,16]$. As the tetrahedral mesh is adaptively refined in the zone of interest, the number of right tetrahedra changes together with the shape measure of the tetrahedra. In this test example, we compare two initial meshes, one comprised entirely by right tetrahedra, and the other with no right tetrahedra (see Figs. 6(a) and 7(a)).

For the purpose of element selection, we presume here the error function

$$
\operatorname{Error}(P)=\frac{1}{0.0001+d^{2}},
$$

where $d$ is the distance from each point $P$ to the corner. We get an error per element as usual, by integrating the function Error in each tetrahedron $t$. Here, we suppose a linear approximation of the function Error at the vertices in each tetrahedron, so that the error per element, $E(t)$, is equal to the volume of $t, \operatorname{vol}(t)$, multiplied by the value of the function at the barycenter $B_{t}$ of $t$ :

$$
E(t)=\operatorname{vol}(t) \cdot \operatorname{Error}\left(B_{t}\right) .
$$

Since $E(t)$ is weighted by the volume of the element, the formula (4) includes the scale of the mesh in the vicinity of the singularity. Figs. 6 and 7 show the two initial and final meshes after ten local refinements. 

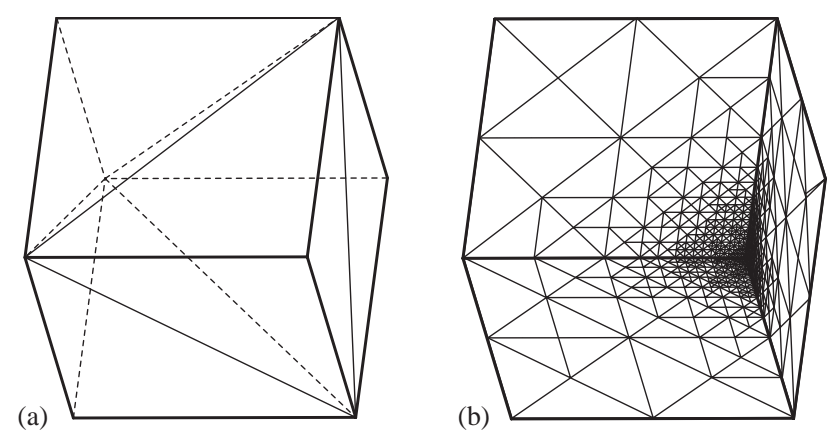

Fig. 6. Three-dimensional local refinement, with no right tetrahedra in the initial mesh: (a) initial mesh, five no-right tetrahedra and; (b) final mesh, 10018 tetrahedra and 2048 nodes.
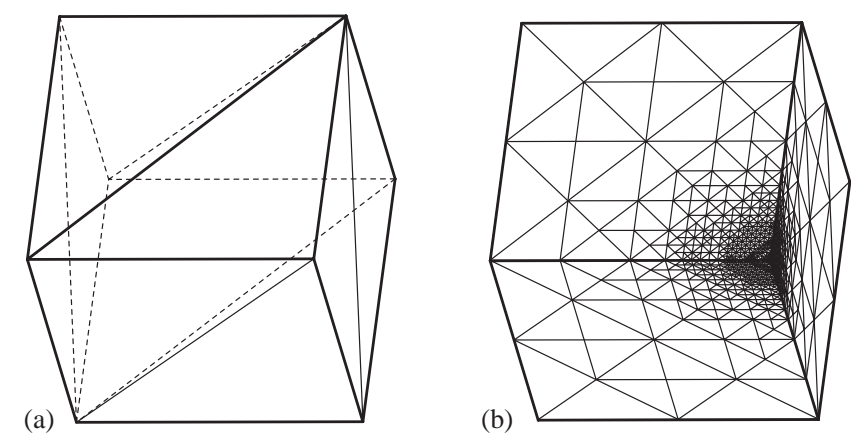

Fig. 7. Three-dimensional local refinement, with right tetrahedral initial mesh: (a) initial mesh, six right tetrahedra and; (b) final mesh, 13441 tetrahedra and 2727 nodes.

Fig. 8(a) shows the evolution of the relative number of right tetrahedra (quotient between the number of right tetrahedra and the total number of tetrahedra) and the relative volume covered by right tetrahedra for this problem when the initial mesh contains six right tetrahedra. The evolution of the same relative numbers, when the initial mesh contains five non-right tetrahedra, is shown in Fig. 8(b).

The evolution of the shape measurements through the sequence of ten refinements for this test case can be observed in Fig. 8(c). The minimum value of $\eta$ has been used for this purpose. Better results in shape measure could be obtained by using some appropriate mesh improvement techniques like swapping or smoothing or local transformations. However, it should be noted that although initially the right tetrahedral mesh presents inferior shape measure, the minimum $\eta$ remains unchanged from the second local refinement at a value that is higher that the values obtained from a non-right tetrahedral initial mesh represented by the dotted line in Fig. 8(c).

\section{Conclusions}

In this paper, we have proved that the 8-tetrahedra longest-edge (8T-LE) partition of a right-type tetrahedron yields into a sequence of right-type tetrahedra. At most, only three different similarity classes 


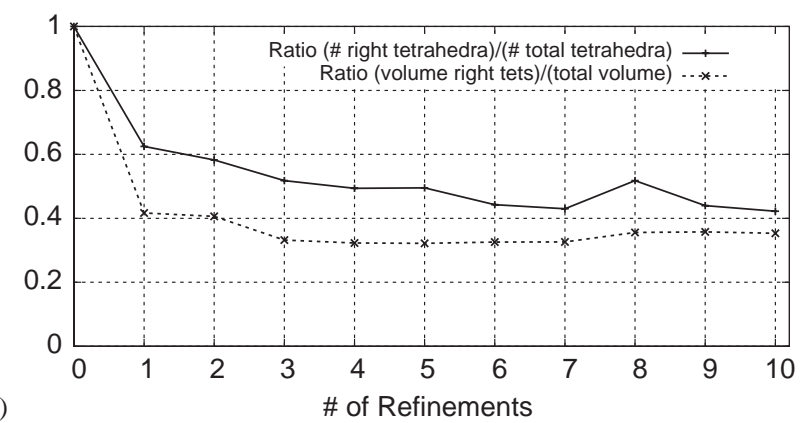

(a)
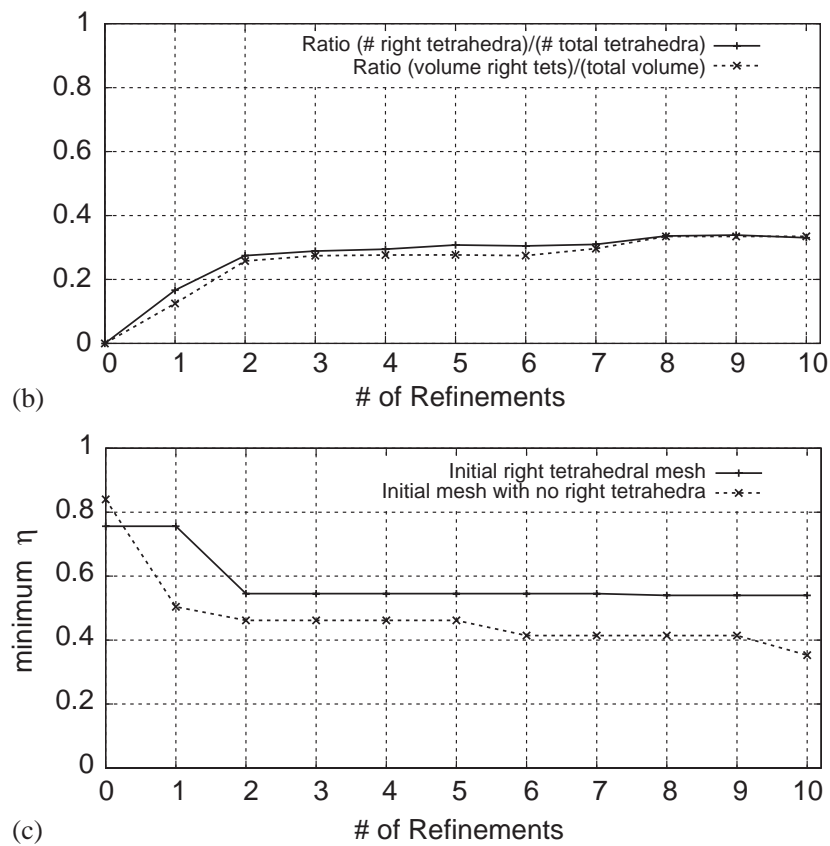

Fig. 8. Number of right tetrahedra and evolution of shape measure: (a) evolution of relative numbers of right tetrahedra; initial mesh of right tetrahedra; (b) evolution of relative numbers beginning with no-right tetrahedra; (c) evolution of minimum shape measure $\eta$ for these initial meshes.

are obtained by the iterative application of the 8T-LE partition to any initial right-type tetrahedron. Hence, this sequence satisfies the maximum angle condition. The non-degeneracy property of these meshes is simply deduced. Acute type mesh, maximum angle condition and non-degeneracy are convenient properties in finite element analysis.

\section{Acknowledgements}

This work has been partially supported by Project UNI-2003/16 of the University of Las Palmas de Gran Canaria, and by Project PI-2003/35 of the Gobierno de Canarias. 


\section{References}

[1] O.C. Zienkiewicz, Y.C. Liu, G.C. Huang, Error estimation and adaptivity in flow formulation for forming problems, Int. J. Numer. Methods Eng. 25 (1988) 23-42.

[2] O.C. Zienkiewicz, J.Z. Zhu, N.G. Gong, Effective and practical h-p version adaptive analysis procedures for the finite element method, Int. J. Numer. Methods Eng. 28 (1989) 879-891.

[3] J. Ruppert, R. Seidel, On the difficult of triangulating three-dimensional nonconvex polyhedra, Discrete Comput. Geom. 7 (1992) 227-253.

[4] P.L. George, F. Hecht, E. Saltel, Automatic mesh generator with specific boundary, Comput. Methods Appl. Mech. Eng. 92 (1991) 269-288.

[5] W.F. Mitchell, A comparison of adaptive refinement technique for elliptic problems, ACM Trans. Math. Software 15 (4) (1989) 326-347.

[6] I. Babuška, A.K. Aziz, On the angle condition in the finite element method, SIAM J. Numer. Anal. 13 (2) (1976) $214-226$.

[7] M. Kř́̌žzek, On the maximum angle condition for linear tetrahedral elements, SIAM J. Numer. Anal. 29 (2) (2001) 513-520.

[8] M.C. Rivara, Mesh refinement based on the generalized bisection of simplices, SIAM J. Numer. Anal. 2 (1984) $604-613$.

[9] A. Plaza, G.F. Carey, Refinement of simplicial grids based on the skeleton, Appl. Numer. Math. 32 (2) (2000) $195-218$.

[10] R. Schneiders, Octree-based hexahedral mesh generation, Int. J. Comput. Geom. Appl. 4 (2000) $383-398$.

[11] M.A. Yerry, M.S. Shephard, Automatic mesh generation by modified-octree technique, Int. J. Numer. Methods 20 (1984) 1965-1990.

[12] I.G. Rosenberg, F. Stenger, A lower bound on the angles of triangles constructed by bisecting the longest side, Math. Comput. 29 (130) (1975) 390-395.

[13] M.C. Rivara, G. Iribarren, The 4-triangles longest-side partition of triangles and linear refinement algorithms, Math. Comput. 65 (1996) 1485-1502.

[14] A. Plaza, J.P. Suárez, M.A. Padrón, S. Falcón, D. Amieiro, Mesh quality improvement and other properties in the fourtriangles longest-edge partition, Comput. Aid. Geom. Design 21 (4) (2004) 353-369.

[15] A. Liu, B. Joe, Quality local refinement of tetrahedral meshes based on bisection, SIAM J. Sci. Statist. Comput. 16 (1995) 1269-1291.

[16] A. Plaza, M.A. Padrón, G.F. Carey, A 3D refinement/derefinement combination to solve evolution problems, Appl. Numer. Math. 32 (4) (2000) 285-302.

[17] A. Plaza, M.C. Rivara, Mesh refinement/derefinement based on the 8-Tetrahedra longest-edge partition, in: Proceedings of the 12th International Meshing Roundtable, Santa Fe, NM, 2003, pp. 65-78.

[18] S. Korotov, M. Kř́ízek, Acute type refinements of tetrahedral partitions of polyhedral domains, SIAM J. Numer. Anal. 39 (2) (2001) 724-733.

[19] M. Křížek, L. Qun, On diagonal dominance of stiffness matrices in 3D, East-West J. Numer. Math. 3 (1995) 59-69.

[20] I. Kossaczký, A recursive approach to local mesh refinement in two and three dimensions, J. Comput. Appl. Math. 55 (1994) 275-288.

[21] A. Liu, B. Joe, On the shape of tetrahedra from bisection, Math. Comput. 63 (1994) 141-154.

[22] A. Liu, B. Joe, Relationship between tetrahedron shape measures, BIT 34 (1994) 268-287.

[23] J. Dompierre, P. Labbé, F. Guibault, R. Camarero, Benchmarks for 3D unstructured tetrahedral mesh optimization, in: Proceedings of the Seventh International Meshing Roundtable, Sandia Report SAND 98-2250, 1998, pp. $459-478$.

[24] V.N. Parthasarathy, C.M. Graichen, A.F. Hathaway, A comparison of tetrahedron quality meshes, Finite Elements Anal. Design 15 (1993) 225-261.

[25] M. Bern, D. Eppstein, Computing in Euclidean Geometry, 2nd Edition, in: D.-Z. Du, F.K. Hwang (Eds.), World Scientific, Singapore, 1995, pp. 47-123.

[26] S.H. Lo, 3D anisotropic mesh refinement in compliance with a general metric specification, Finite Elements Anal. Design 38 (2001) 3-19. 